\title{
226 TREATMENT AND REHABILITATION OF THE CRITICALLY ILL PATIENT ON THE INTENSIVE CARE UNIT (ICU) - THE ROLE OF IMPAIRED MICROCIRCULATION
}

\author{
*Karl Werdan, *Diethelm Kühnert, **Ursula Mueller-Werdan, *Sebastian Nuding \\ ${ }^{*}$ Department of Medicine III, University Hospital , Halle (Saale), Germany, ${ }^{* \star}$ Charité - \\ Universitätsmedizin Berlin and Protestant Geriatric Center Berlin, Berlin, Germany.
}

Introduction: Impaired microcirculation contributes substantially to mortality of ICU patients and to prolonged rehabilitation.

Purpose: To describe microcirculatory impairment in critically ill patients and its consequences for treatment and rehabilitation.

Methods: Narrative review and presentation of preliminary data from the MicrocircMODS trial.

Results: Microvascular dysfunction contributes to a variety of disease states by triggering inflammation, thrombosis and fibrosis (1-3). Noninvasive optical techniques (e.g. Microscan) allow visualization and quantitative analysis of the sublingual microcirculation in patients at the bedside (4-6). The percentage of perfused small vessels $(<20 \mu \mathrm{m})$ is lower e.g. in patients with severe sepsis (2), heart failure and even less in patients with cardiogenic shock $(92 \%$ vs. $63 \%$ vs, $49 \%$; p $<0.001)$; with a higher proportion of perfused vessels in surviving patients (3). Topical application of acetylcholine reverses microvascular impairment (3) documenting - in principle - reversibility of these alterations. No medication is available which specifically attenuates microcirculatory dysfunction.

In the ongoing monocentric prospective MicrocircMODS-trial we try to improve impaired microcirculation of patients with multiple organ dysfunction syndrome (MODS) with a biorhythmdefined physical stimulation of impaired spontaneous arteriolar vasomotion by an electromagnetic alternating field of low magnetic flux densitiy ("Physical Vessel Therapy BEMER ${ }^{\circledR}$ ), which has been shown to reduce disturbed microcirculation in diabetic patients (7). In the MicrocircMODS-trial 10 patients with MODS are to be treated for four days - three times a day - for 8 min each with physical stimulation, with a flow density of 10.5 to $21 \mu \mathrm{T}$. Primary end point is the effect on impaired sublingual microcirculation. In April 2018 the last patient has been included, and 6-month-survival rate will be obtained in October 2018.

Conclusion No pharmacological approach is yet at hand to improve impaired microcirculation of ICU patients. A positive result of the MicrocircMODS trial could lance a new physical concept of treatment and rehabilitation of ICU patients.

References: 1) Circulation 2016;118:157; 2) Crit Care Med 2013;41:791; 3) Am Heart J 2004;147:91; 4) Crit Care 2012; 16:224; 5) Crit Care 2015;19:388 6) Crit Care 2016;20:35;

7) Z Gerontol Geriat 2013;47:415.

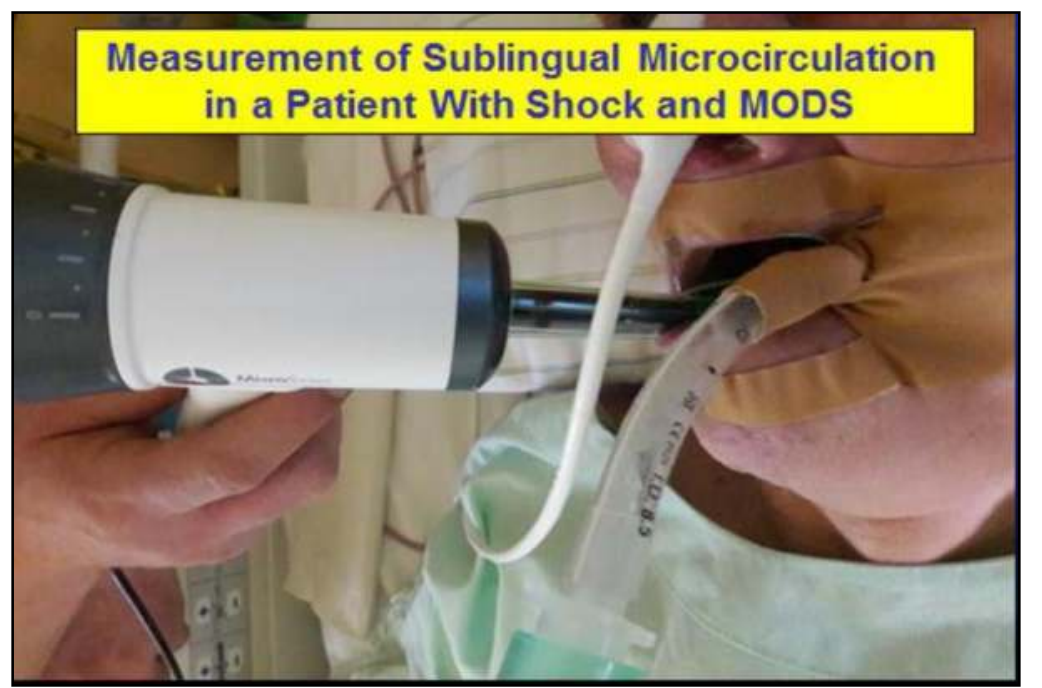

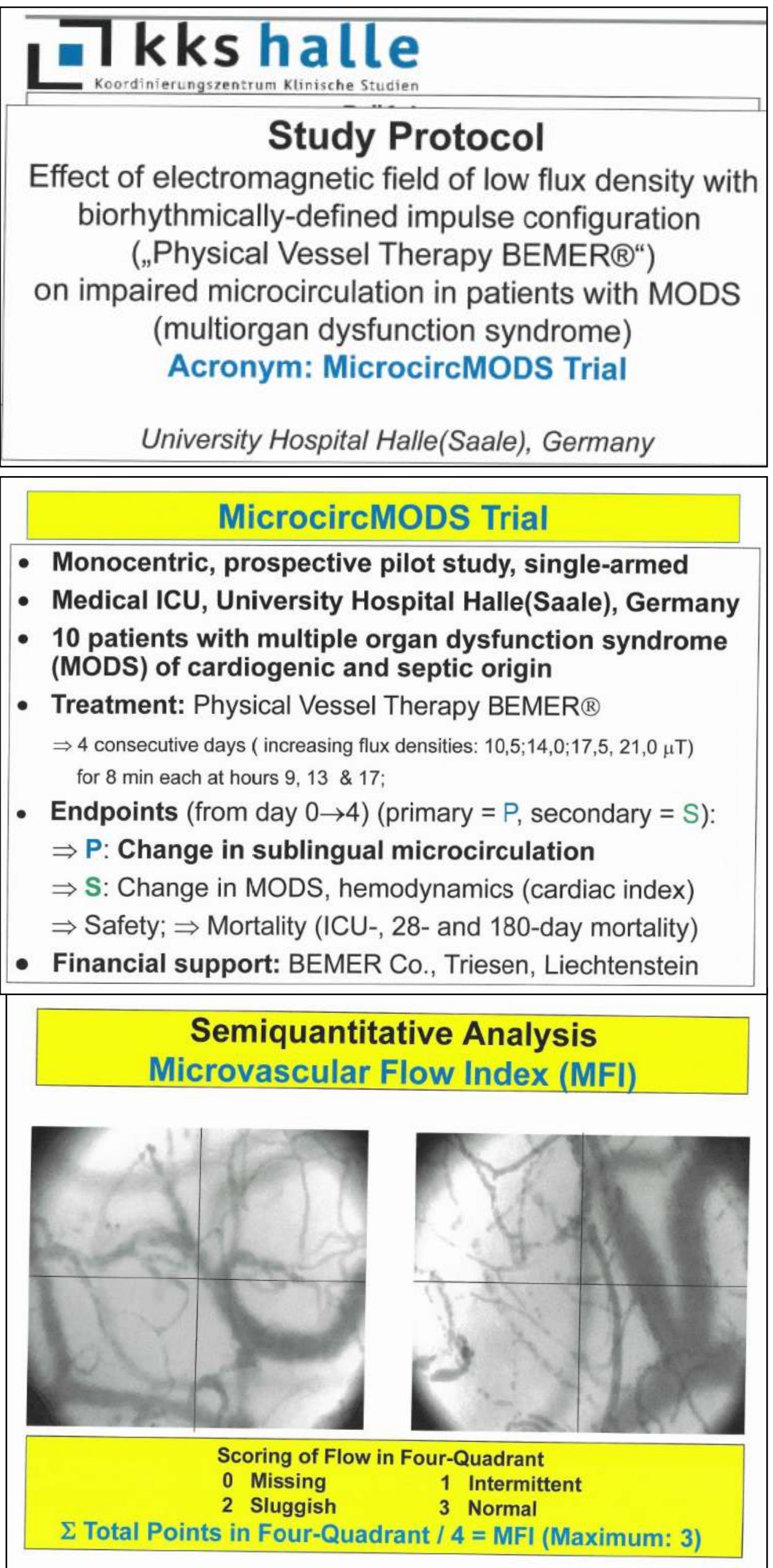
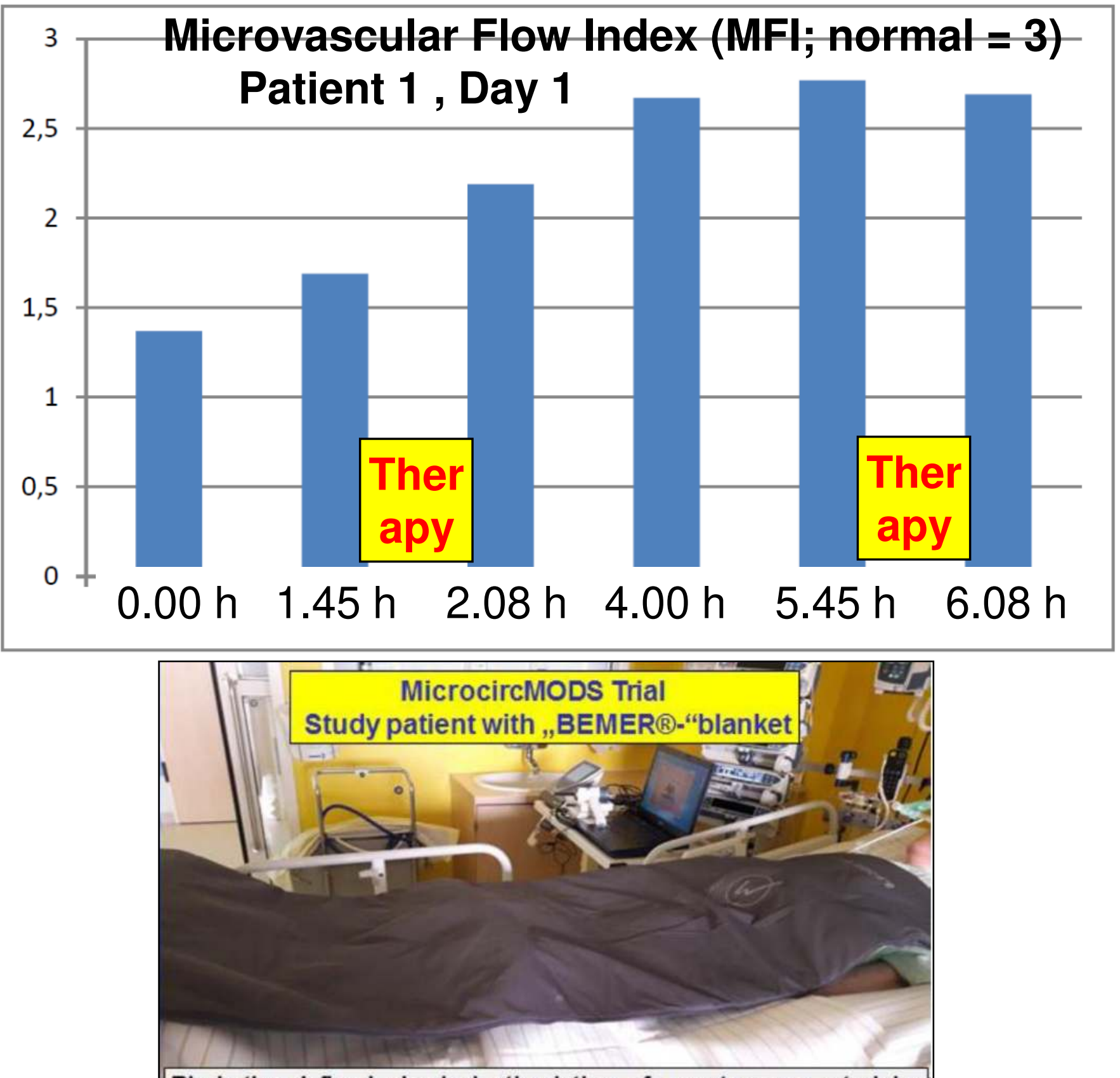

Biorhythm-defined physical stimulation of spontaneous arteriolar vasomotion by an electromagnetic alternating field of low magnetic flux densitiy (,Physical Vessel Therapy BEMER $\circledast^{c 4}$ 